\title{
The Journey towards Successful Research in a Diabetes Clinic: Expectations vs. Reality
}

Hayat Mushcab ${ }^{1 *}$, W George Kernohan ${ }^{1}$, Jonathan Wallace ${ }^{1}$, Roy Harper ${ }^{2}$ and Suzanne Martin ${ }^{1}$

${ }^{1}$ Institute of nursing and health research ,Ulster university, northern Ireland, uk

${ }^{2}$ Ulster hospital, southeastern health and social trust

\begin{abstract}
In theory, conducting robust clinical research is a matter of developing a strong proposal with ethical governance and following through the complete research cycle. However, the reality of design and conducting research is a complicated process involving many opportunities and obstacles that challenge the researcher from start to finish. In this paper we reflect on our experience of conducting our study and how these were responded to and influenced the research. A brief overview of our study's design and location is provided followed by a pragmatic discussion of the challenges as they arose. An understanding emerged of the critical need to nurture a mutual understanding and strong collaboration between the research team, clinical team and the patient as a key facilitator in completing a research trial.
\end{abstract}

\section{Introduction}

"If we knew what it was we were doing, it would not be called research, would it?” - Albert Einstein.

Diabetes is one of the leading threats to health globally; in 2010, 300 million people worldwide were living with diabetes, and 344 million others were at risk of developing Type 2 Diabetes (T2D) [1,2]. T2D is prevalent worldwide. Recent research has been focusing on the atrisk population to prevent or delay the development of their diabetes by lifestyle interventions or some medications in addition to exploring how to support individuals living with T2D self-manage the condition [3]. Clinical trials are regarded as the most robust type of evidence to help inform the policies and practices of the healthcare system [3]. In order to achieve optimal illness prevention, disease diagnosis or treatment options for patients with a particular medical condition, clinical trials are conducted [4]. They can involve an individual person (studies of " $n=1$ "); a group of people, a specific human behavior or tests upon tissue samples [5].

Empirical studies with commonly acknowledged methodological designs are used to test the efficacy of a new medicine or to determine if a new therapeutic solution makes a difference in patient treatment [3]. Even though clinical trials are the key to scientific discovery and improvements in the medical field that deliver evidence to healthcare systems around the world, the link between them and medical progress is frequently misunderstood [3]. Experts from user groups, the government, the academic research field, pharmaceutical companies, biotechnology and medical devices industries and nonprofit research networks have made clinical research their main focus in UK and all these parties, combined together are components of continuous quality improvement in the UK's health economy [6]. However, time and cost of conducting research are major challenges that threaten clinical research [6]. It is crucial to highlight and reflect upon these challenges to progress towards more successfully managed and completed robust clinical trials [7]. Within the real world setting, achieving this requires organizational and personal attributes of all the 'players' to be considered, for example amongst research organizations, industry managers, scientists, academics, clinicians, patients and any entity involved in a clinical research project [6].

In theory, designing and conducting a trial of a specific intervention can be described in terms of a pathway or journey and could be considered a straightforward process that starts with a thoroughly researched proposal, aiming towards a positive clinical outcome upon completion. However, in reality, researchers face many obstacles and challenges throughout the design and delivery of research trials $[3,8]$. This process can be very time consuming; years can pass from the moment an idea was conceived to the initiation of the trial to completing it and disseminating findings [3]. The obstacles include: finding sufficient funds, the complexity of obtaining ethical and regulationary approval, establishing the clinical trial in the proper organization, administering comprehensive yet easily understood consent forms, patients recruitment, data collection, analysis and completing large amounts of paperwork [3]. Challenges in clinical research in the UK have been categorized into four main areas: (a) Regulations and governance, (b) Knowledge and expertise, (c) Networks and strategy and (d) Incentives and drivers [6]. These broad areas will be used to guide this paper. A clinical researcher's long-term aim is to improve the healthcare provided by the clinicians and received by individuals [9]. Therefore, a proposal has to be well established and thoroughly documented with comprehensive methods for the study design and data analysis creating a clear and strong infrastructure [3, 4]. A robust study design that delivers valuable results has to have sufficient sample size, statistical power and bias control [4]. It also has to be achievable in the proposed clinical setting. In our research, a three-arm Interrupted Time Series (ITS) design was developed to determine the optimal use of connected health technology to achieve self-management of T2D. ITS design was considered to be the most appropriate method to explore a complex intervention and has been shown to be useful within the context of a health service provision with monitoring of patients on a routine basis [10]. Connected health technology is defined according to the World Health Organization as: "The delivery of health care services, where distance is a critical factor, by all health care professionals using information and communication technologies for exchange of valid information" [11]. In order to demonstrate an effect, the required sample size was 80 patients living with T2D undertaking insulin

${ }^{*}$ Corresponding author: Hayat Mushcab, Institute of Nursing and Health Research, Ulster University, Northern Ireland, United Kingdom, Tel: +447460314350; E-mail: hayat.mushcab@gmail.com

Received August 05, 2015; Accepted September 21, 2015; Published September 28, 2015

Citation: Mushcab H, W George Kernohan, Wallace J, Harper R, Martin S (2015) The Journey towards Successful Research in a Diabetes Clinic:Expectations vs. Reality. J Health Med Informat 6: 204. doi:10.4172/2157-7420.1000204

Copyright: () 2015 Mushcab H, et al. This is an open-access article distributed under the terms of the Creative Commons Attribution License, which permits unrestricted use, distribution, and reproduction in any medium, provided the original author and source are credited. 
treatments, giving the study $90 \%$ statistical power. The trial took place in a district general teaching hospital in Northern Ireland. The research team consists of $\mathrm{PhD}$ student and three professors of Health Research, Innovation, and Occupational Therapy working with an endocrinologist and his team including a diabetes research nurse. The telemonitoring solutions are services currently provided by one Health and Social Care Trust as part of a "Connected Health" contract within Northern Ireland, UK. In March 2011, this contract, valued at $£ 18 \mathrm{~m}$ and designed to manage up to 20,000 patients over a 6 year period was awarded by The Department of Health, Social Services and Public Safety [12].

In this paper we describe our experience of conducting a clinical health technology trial in a Diabetes clinic. We took a pragmatic approach to reflect our experience conducing a study in the diabetes clinic, explore how the experience evolved chronologically and define the difference between our theoretical expectations and the reality of the experience, as these challenges are likely to arise again.

\section{Study Proposal and Methodology Development}

The impact of the trial's result can be significantly affected by the long period starting from when a trial question was addressed to obtaining the answer. If the results come after a long time lag they may even be irrelevant to the clinical practice [3], because an alternative solution may have been found or priorities changed over time. There are some challenges to design a clinical trial in T2D [3]. Having developed the research questions, the dilemma was how best to match this to a suitable study design. Intervention studies in particular are often time consuming and require extensive planning and preparation [9]. In 2006, The Medical Research Council (MRC) developed a guidance document titled (Developing and evaluating complex Interventions: new guidance), which is an update to the original MRC Framework for the Development and Evaluation of RCTs for Complex Interventions to Improve Health that was published in 2000. It is intended to help researchers to choose appropriate methods, extending the coverage to include non-experimental methods and complex interventions [13]. Although randomized controlled trials (RCTs) are considered to be the strongest study design to establish the effect of an intervention or a new medication, quality improvement and public health [14], many consider RCTs to be unsustainable and impractical because of the time consumption, expense involved [3], lack of external validity [9], and overall challenge to implement within the timeframe allocated. Quasi-experimental designs are considered to be a good alternative, particularly when individual randomization is not feasible for clinical or service-delivery reasons [9]. Quasi-experimental designs are increasingly used for the evaluation of clinical and/or practicebased interventions applied under real world circumstances, where individual-level RCT designs are not suitable [14].

Initially, our study was conceived to be a five-armed RCT: testing four types of support against one control arm. The aim was to evaluate four different connected health solutions offered by the diabetes clinic at the hospital. These interventions varied from intensive level of telemonitoring by the clinical team to independent self-management of T2D the patient. However, the proposed sample size and amount of data to be collected was assessed as being unreasonable and this design was deemed too ambitious for available resources and timeframe. There was uncertainty regarding the random allocation of devices, rather than allocation on the basis of clinical need. After a comprehensive literature review using evidence from primary studies, systematic reviews and review of the designs acceptable to the Cochrane Collaboration [15], to inform study design, we decided the
Interrupted-time series (ITS) design -a quasi-experimental design- was most suitable to achieve our objectives. ITS design was introduced to health services research literature by Gillings et al. [16], it is defined as a collection of observations made consecutively in time and each study subject serves as their own control [10], data are collected at multiple occurrences over certain period of time before and after an intervention is introduced to study whether it significantly effected the underlying condition [17]. ITS design can be used to strengthen the before-andafter study designs and plays a pivotal role in assessing the effects of health services and policy interventions because they can account for the pre-intervention level and trend of the outcome measures when several changes are made to patient care "Interruptions" [16,17].

\section{Regulations and Ethics}

The Medicines and Healthcare Regulatory Agency (MHRA) was established to administer the regulatory approval process following the EU Clinical Trials Directive within the UK in 2004. This new process resulted in changes in obtaining regulatory approval. The MHRA is concerned with clinical trials of an Investigational Medicinal Product. Regulatory adherence became a more time consuming process that requires more detailed information, intensive documentation and demonstration of suitability of researchers including Good Clinical Practice (GCP). In 2003 and 2007 an amendment took place in the EU Medical Devices Directives for Medical Devices Research, making it subject to different set of regulations related to marketing and clinical use of these devices [6]. Consequently, the EU Directives changes and the augmented requirements of an academic institution for reviewing multiple aspects of a clinical trial have affected the ease of management and the overall set-up time of a study [3,6]. Thus, it takes (on average) the Research and Development (R\&D) department 114 days, Ethics 91 days and Regulatory 77 days to provide approval from the day of submission. In the last decade, an increment was found in the time required to prepare for, and receive MHRA regulatory approval for a clinical trial. In addition to the time required, the amount and type of documentation has also increased considerably to fulfill ethical review requirements. Adding all these factors made the new process of obtaining approvals a huge burden for researchers with its complexity, time consumption and rigorous documents preparation in order to satisfy regulatory and governance requirements [6]. Moreover, obtaining ethical approval and decision-making process can be delayed where the researcher is not fully aware or absolutely competent in either required process or expectations [3].

Following these many requirements is a serious challenge for not only novice researchers but often changing requirements and amendments to regulation can create delays for even more experienced researcher, this is often considered one of the greatest challenges associated with conducting a clinical trial $[3,6]$, and to avoid the inconvenience, researchers avoid clinical trials completely, even turning to laboratory work [3]. Typically the research design for projects involving medical devices often differs from more standard RCT trials; and it is not unusual for delays in obtaining approvals to occur due to a lack of understanding from ethics committees about what features comprise good research practice when knowledge of device functionality and application has a gap of understanding [6].

Recently, an Integrated Research Application System (IRAS) [18], for gaining NHS permissions, was introduced to the clinical research domain. Even though it was considered extensive and considerably lengthy it was a welcomed introduction by researchers in the UK [6]. It has been acknowledged that the regulatory approvals often embedded with participant recruitment and informed consent whilst unavoidable 
can add significantly to the time consumed as the team moves toward engaging directly with research participants. This process of educating candidates on the clinical trial they are about to take part of and documenting it and potentially gaining their consent to participate in the study is crucial and required [3]. The lengthy process begins with developing a properly worded patient information sheet (PIS), consent form, explaining the documents to the potential participants and research team, obtaining their signature on the documents and ends with securely filing them and tracking the paperwork throughout the patient recruitment process $[3,19]$.

Reflecting on our work, from research design and obtaining ethical approval, the protocol had to obtain four different regulatory governance approvals. It took nearly ten months to obtain approvals from: University Research Governance "filter" committee (for preliminary screening), ORECNI (for ethical approval), AccessNI (for staff clearance) and HSC $\mathrm{R} \& \mathrm{D}$ (for Trust approval). To obtain the university's approval, the protocol was peer reviewed by two different academic members of staff who are part of the research institute (but not part of the research team), and filter committee approval was provided two weeks later. Meanwhile, the researcher completed the (IRAS) online applications needed for submission to the Office for Research Ethics in Northern Ireland (ORECNI) and the formal application to HSC R\&D in the South Eastern Trust. Final ORECNI approval was granted four months from study protocol submission, attending the defense hearing and completing the minor amendments they requested. These amendments included developing three separate PISs for each technological solution used in the study and two different consent forms to be administered with every interruption (Week 0 and Week 12) each with three signatures; the patient's, the physician's and the researcher's.

While awaiting the final Ethical approval, a criminal history disclosure (AccessNI) was needed by the HSCR\&D office in the trust to enable them to grant the researcher an honorary contract. This requirement of the honorary contract is embedded within the research allowing them to establish the study in their hospital. Some employers in Northern Ireland must check a person's criminal history before recruiting them to any position, in this case the AccessNI approval aims to protect patients and their personal data [20]. The first letter from AccessNI was issued within a few weeks but was never received. The researcher then had to submit a new application and await another letter to be issued which caused a long delay in the commencement of the trial. Finally, an honorary contract for the duration of the study was granted to the researcher allowing her to interact with patients and obtain patient data.

\section{Recruitment}

Recruitment is often the most challenging aspect of conducting a trial. It can "make or break" a research study [21]. A clinical trial cannot be successfully completed without the required number of participants fitting the predetermined criteria. At the same time, research involving human subjects within the health service has become a progressively complex environment and challenging to operate within [3].

Patient recruitment and retention in a clinical trial depend on three factors: patient-related, healthcare provider-related and systemrelated [3,22-24]. The key to successfully overcome these challenges is flexibility and adaptation to recruitment issues as they arise [21] while avoiding alterations in the inclusion and exclusion criteria, which will potentially reduce the validity of the findings [23].

We conducted a multiple step participants' enrollment strategy to facilitate the recruitment process. Before every clinic, the researcher - who became an honorary NHS employee - would scan the scheduled patients for potential participants who meet the inclusion criteria. The researcher would approach them while waiting for their appointment and introduce the study to them providing them with sufficient information about the study, the different technological solutions used in the trial that might interest them and the duration of the study. Then the researcher would notify the physician of the patient's interest. The physician would meet the patient and have a further discussion to decide if the technology would be beneficial for the patient. Once the patient has agreed to take part in the study, formal consent was obtained and patient is referred back to the researcher. Even with careful planning and multiple steps, patient recruitment was challenging.

\section{Patients-related challenges}

Each person who met the inclusion criteria was invited to join the study, but each one presented with unique circumstances, when it comes to participation. People living with diabetes for many years, tend to adapt to the lifestyle of their condition and they may feel more in control and comfortable with their standard management methods; they may be reluctant to change. Whereas newly diagnosed patients have a level of vulnerability with the diagnosis. Those are more likely to enter a clinical trial with high hopes of curing their disease [3]. T2D patients vary in age from adolescents to the elderly, and sometimes children. Our study criteria included patients with T2D, adults (above the age of 18) and undertaking insulin treatment. Most of our participants were elderly; and some of them do not fully understand nor completely trust the medical establishment [24]. From our experience, some older patients prefer a face-to-face human contact as they are not accustomed to using technology in their daily lives, and hence are unaccustomed to technology as a way to support the management of their illness.

A very common issue that occurs in most clinical trials is the burden of clinical visits required by the study protocol. Patients taking part in the study may find participation to be costly and time consuming [3]. Through planning the study around their normal attendances, this issue was eliminated in our study design. Normally, for an unstable patient, or patients who needs monitoring, they are scheduled a three monthly clinic visit and we did not change that for our trial. Another matter that raised a challenge was participants' general knowledge of clinical trials and research. Patients do not normally have sufficient knowledge about research and thus need intensive information to make informed choices about taking part of a trial, especially if the trial was lengthy and therefore, physical limitations like traveling and transportation will occur [22]. The study protocol suggests that the researcher briefs the patient before meeting the physician about the trial, the different solutions and the length of the study, providing the patient with comprehensive Information sheet about each technology included in the trial. The physician will decide on individually designed care plan for each patient and if suitable and advised, they will elaborate and give the patient more information about the study to help them decide and consent for taking part and data will be collected from patients every three months.

The extensive paperwork associated with collecting data including the consent form and questionnaires can be burdensome to the participants [3]. Instead of troubling the participants with this amount of paperwork in the clinic, we gave them the option to complete the questionnaires at home and post or email them back to the researcher at the university. Most of these questionnaires were sent back to the researcher; however, a few participants have failed to do so. 
Socioeconomic status is a vital factor that can affect a person's level of engagement with the healthcare system and exposure to clinical trials. A patient with higher socioeconomic status (i.e. income, education and occupation) is more likely to be aware of the clinical research opportunities available and better equipped to weigh the risks and benefits of participating in a trial [3].

Finally, some patients have strong preferences about receiving or avoiding one of the study interventions, and it is their right to refuse participating in a trial. Or in other words, some patients are uncomfortable with being, as they see it, "guinea pigs". To address this fear, the ITS design allows the physician to retain control over the interventions, and so the technology is part of patient care: only the evaluation is additional.

Nonetheless, all patients who were satisfied and pleased with the results of these solutions had the option of continuing using the technology at the end of the trial. The clinical team continued with the standard follow-up procedure, which is for the patient to be seen every three to six months.

\section{Healthcare providers-related challenges}

Healthcare providers' knowledge, attitudes and beliefs about clinical trials may drive them to approach patients about research. Thus, healthcare providers play a critical role in the recruitment process [24]. However, physicians face barriers conducting trials due to lack of adequate time to discuss the study with their patients, limited research resources and/or over loaded staff $[22,24]$. Patient recruitment in a clinical trial is considerably higher when the patient believes in the physician's integrity [23], thus, healthcare providers that are research enthusiasts are often the ones introducing the option of trial to the patient $[3,24]$. The relationship between the research team and the patient is a key to successful recruitment and retention of participants. This special connection is nourished and sustained mainly with the physician's support and encouragement [3]. Moreover, physicians worry that a trial may cause excessive hardship to older patients who already experience declining functional status; therefore they normally hesitate from recruiting older adults to a clinical trial [24].

Our study protocol does not interfere with the physician's decision on the individually designed treatment plan. The physician decides, in agreement with the patient, if they are ready to try the technology to self-manage their condition. Only then, the physician will discuss the study with the patient and explain the advantages of taking part in the study. Recruiting patients to the technology may add extra work; as the physicians need to register the patient onto the system and remotely monitor the patient's readings on a weekly basis. A number of physicians were reluctant to recruit cases into the study and telemonitoring solutions in general for a number of reasons. 1) They did not believe in the advantages the technology might provide to the patient, 2) They were avoiding any burden it may cause them, or simply 3) They were not interested in taking part of the study. To help address this and in her honorary role as a Trust staff member, the researcher was given access to the telemonitoring systems to help in the time consuming registration and facilitate the administration process.

\section{System-related challenges}

Difficulties in healthcare system and the hospital system particularly can contribute to poor recruitment of study participants no matter what group or age they are. Challenges like limited resources, funding or even the introduction of the "paperless environment" can be problematic for both patients and research team and can affect the trial's progression. Additionally, disinterest in the research among the administrative staff can occur and that may stem from their lack of research training and knowledge level and can result in problems with recruitment, and retention of study participants to clinical trials [24]. During our study, we faced several system-related challenges.

Firstly, in order for the researcher to establish the trial in the hospital, they had to obtain an honorary contract with the trust to be authorized to have contact with patients, access their confidential information and be able to properly conduct a trial. The process took several weeks until the Access NI clearance came through to be able to finalize the contract.

Secondly, the first step of the recruitment process was to scan the database of potential participants that fit the proposed criteria before every clinic. Therefore, computer access for the researcher was needed. A request was submitted to the IT department by the endocrine consultant who is the chief investigator (CI) in our trial.

Thirdly, while the trial is running and patient recruitment is still in progress, the hospital moved from manual check-in for outpatients done by a receptionist, to electronic self-check-in using kiosks installed in the departments. While this is an advanced step towards paperless environment that is intended to take some of load off the staff and save time for both staff and patients, the transition from the old system to the advanced computerized system needed effort from staff and patients to adapt and adjust to it. This took some time and caused some delays in patients appointments and therefore there was little time to introduce the study during the transition. A few weeks later, the system was picked up quickly and ran smoothly.

Finally, the simplest yet most difficult challenge to overcome is the limited available funding to provide one of the most popular telemonitoring solution amongst physicians and desired by patients because of its ease of use giving the patient more control over their condition. Currently, the NHS is going through the biggest financial deficit in its history, and the increasing demand for care is causing huge pressure on services provision [25]. This issue has restricted the physicians in their patient selection to register to the technology, thus, slowed the recruitment process significantly.

Approximately 45 patients visit each diabetes clinic held by our clinical team. In 13 months of recruitment, we were able to introduce the study to 166 potential participants that fit our criteria in average of 4.3 patients per clinic in total of 39 clinics attended. These patients are on average of 63 years of age $(S D=10.6)$ and had $\mathrm{T} 2 \mathrm{D}$ diabetes for nearly 13.5 years. $17 \%$ of the screened patients consented to take part. $11 \%$ of them did not have the clinical team's recommendation to use the technology to manage their condition for various reasons, such as patients do not show intentions for commitment, patient is too unstable or they suffer from other health complications. Equally, $11 \%$ of the patients were not interested in using technology: $7 \%$ had good management of their T2D and were content with the standard care they are receiving. $4 \%$ did not need any telemonitoring solutions because they recently stopped taking insulin treatments. Another 3\% needed a carer to help them in their daily life. $18 \%$ of the patients gave no answer or final decision whether to proceed with the study or to decline. However, the majority (29\%) of the patients used -or currently using - telemonitoring solutions to manage their T2D where for the study we required them to be naïve to using technology (Figure 1).

\section{Data Collection and Management}

Data collection is the cornerstone of clinical trials and turning this data into meaningful information is the core purpose [26,27]. 
Having the correct research question from the beginning will enable the researcher to gather the appropriate variables [28] that answer their proposed question [29]. A variable can be defined as: a unit of data for which the definition, identification, representation, and permissible values are specified through a set of attributes [29]. Data collection allows better analysis for a new healthcare plan and it provides scientific and clinical evidence that help assessing the advantages and disadvantages of implementing a new medication or intervention, which empowers the ethics and regulatory agencies to make the right decision when approving it as well as the physicians worldwide when designing a robust care plan $[26,27]$. Even though it is a vital part of a trial, clinicians find the additional burden of data collection to be overwhelming, time consuming and problematic [27,28].

Often, clinical trial protocols can be complicated and challenging for data collection because the clinical team find it difficult to follow them, which could result in missed assessments [27]. Therefore, high-quality data availability for early review and rapid decisionmaking can only be ensured with a well-designed and workfloworiented protocol [26]. Investigators use the study protocol as a map from which data collection follows and enables them to thoughtfully produce the data collection tools before starting the data collection process. Gathering a sufficient amount of data is necessary to have statistical power to determine the efficacy of the new intervention [28]. Interviews and questionnaires are the most common methods of data collection in clinical research. Questionnaires are generally a more efficient, uniform and cheaper way to ask simple questions [30]. Questionnaire development, however, can be a real challenge; it is a task for experienced investigators with questionnaire construction and familiar with data collection and analysis methods [31].

It is important for the patient to understand the importance of data collection and how crucial their compliance is to the trial [27]. However, patients often prefer not to be burdened with completing questionnaires or assigned extra hospital visits to fulfill the data collection requirements [31]. The extensive paperwork associated with collecting data including the consent form and questionnaires can be burdensome to the participants and can limit participation [3]. Thus, the number of data collection tools used in one visit should be minimized to prevent excessive load on the participant. Using numerous data collection tools will also increase the investigator's workload and will contribute to poor patients' recruitment significantly [30].

For our study, we used two types of data collection tools: 1) the hospital patient records to collect their clinical outputs on every visit including (HbAlc, Blood Glucose, Body weight and Cholesterol Levels), 2) questionnaires that were already developed and validated by experts in the field of telemonitoring and diabetes. The protocol was designed to see the participant three times in a six-month period. These visits started with the participant's first visit to the diabetes clinic where they consented to take part in the study and then three questionnaires were administered 1) patient satisfaction, patient quality of life and 3) patient's self-management assessment. Three months later the patients returned for a follow-up visit and we would consent them again for entering phase two of the study. The same measurements taken previously would be taken again in addition to a questionnaire that evaluates the technology in hand. Finally, three months later, we repeat all of that with one additional questionnaire that evaluates the patient's satisfaction with change in the care plan. Instead of troubling the participants with this amount of paperwork in the clinic, we gave them the option to complete the questionnaires at home and post or email them back to the researcher at the university.
Most of these questionnaires were sent back to the researcher; however, few participants have failed to do so.

Managing all the follow-up visits according to which patients are to be seen throughout the study is another challenge a researcher must face. Follow-up process can map data collection in less cumbersome but no less useful ways [31]. Therefore, the researcher in our study had a log of all the participants, with the dates of their every meeting. A followup visit is usually scheduled right after they leave the clinic. If a patient missed their appointment, the researcher would reschedule them for the next clinic and post them a letter to notify them, giving them a four weeks window before withdrawing them from the trials. Collecting data for each participant in a clinical trial efficiently and accurately and according to the study objectives is essential for regulatory compliance, as well as the success of the research effort.

For privacy and confidentiality purposes, each study participant is linked to their information with a unique identifier. Participants' confidentiality is essential for the study's integrity and legalization. Participants' sensitive data are kept in a locked and protected location. Digitally gathered data are stored and backed up in a passwordprotected computer. Participants' data are regarded as highly valuable as collection took serious planning, care, attention to detail and hard work to collect it, repeating it would be difficult, inconvenient, time consuming and expensive and therefore it has to be kept safe.

\section{Conclusion}

Clinical research in the field of type 2 diabetes is facing many challenging scientific issues [31]. However, Conducting a clinical trial is an interesting, exciting and essential mission that is likely to provide new knowledge and improve evidence-based practice and healthcare delivery nationwide. In reality, conducting a clinical trial is not an easy task, and it can be extremely frustrating. The bigger and more complex the research problem, the harder and challenging it gets. It is a major responsibility to deal with patients and be able to meaningfully describe people's lives, minds, feelings and realities with the data they have provided. Human factors make or break any good research effort and scientific knowledge is not always the most important or relevant type of information when dealing with people [3].

While there can be no definitive tools and guidelines to assist a researcher who is about to embark on a new study. This paper is meant to give an idea of what reality looks like for a clinical researcher and

\section{Recruitment Process Statistics}

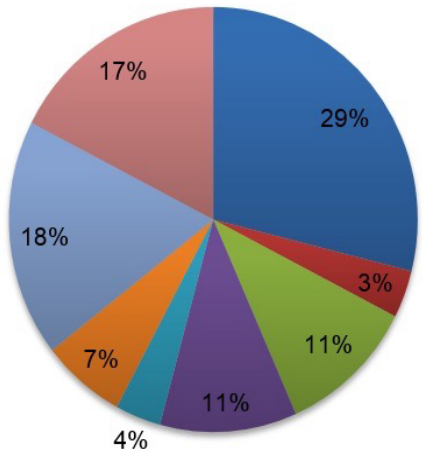

- Has experience with technology - Need Carer

- Doctors did not recommend the technology - Not interested

- Off Insulin treatment

- Has good management

- No reply

m Consented

Figure 1: This pie chart shows the status of patients screened in the recruitment process. Of 166 potential participants only $28(17 \%)$ took part in the study after 13 months of recruitment. 
Citation: Mushcab H, W George Kernohan, Wallace J, Harper R, Martin S (2015) The Journey towards Successful Research in a Diabetes Clinic:Expectations vs. Reality. J Health Med Informat 6: 204. doi:10.4172/2157-7420.1000204

that they have to adapt to every challenge they face and deal with it individually in order to make the best out of a situation to safely overcome these challenges [32,33].

In this paper we broadly cover some of the challenges we faced while conducting our trial that aims to evaluate different levels of telemonitoring solutions and to achieve self-management. The first lesson we learned was that asking the research question is important, but getting the clinical team and patients on board at the start and all the way to the end is essential. Clinical trials need constant dedication from physicians and patients who are willing to participate for the sake of enriching the knowledge and providing better healthcare opportunities for future patients.

\section{Acknowledgement}

The authors wish to thank the team at Ulster Hospital for their enthusiasm for our project and their valuable contribution to the recruitment process $\mathrm{Dr}$. Ursula Brenan MD., Dr Fredrick McElwain MD., Dr James Crothers MD., Dr Paula McDonald MD., Mrs. Lynne Carroll - Nursing Auxiliary, Mrs. Jacquie Wilkinson Diabetes Specialist Nurse, Mrs. Rosemary Donnelly - Clinical Pharmacist,Sharon Carr - Clinical Trial Research Nurse, and receptionists Mrs. Lisa Thomson and Miss Leah Crow.

The researcher would like to express her sincere appreciation to Prof Vivien Coates for her support and valuable contribution to the project and Dr Rana AbuHuwaij for her insightful input and continuous support.=

\section{References}

1. World Health Organisation (2010) "Global status report on noncommunicable diseases". Geneva.

2. JE Shaw, RA Sicree, P Z Zimmet (2010) "Global Estimates of the Prevalence of Diabetes for 2010 and 2030,". Diabetes Research and Clinical Practice 87: 4-14.

3. Institution of Medicine (2010) "Tranforming Clinical Research in the United States: Challenges and Opportunities: Workshop Summary".The National Academies Press, Washington, DC

4. National Research Council (2001) "Small Clinical Trials: Issues and Challenges". The National Academies Press, Washington, DC.

5. NIH: Eunice Kennedy Shriver National Institute of Child Health and Human Development (2012) "Clinical Trials \& Clinical Research".

6. J Swan, M Robertson, S. Evans (2009) "Managing Clinical Research in the UK: Evidence on the challenges of conducting clinical research projects in the UK". The University of Warwick, Coventry.

7. H Murillo, A Reece, R Snyderman, N Sung (2006) "Meeting the Challenges Facing Clinical Research: Solutions Proposed by Leaders of Medical Specialty and Clinical Research Societies,". Academic Medicine 81: 107-112.

8. R Barnes, L Edmunds, S Ward (2008) "Reality of undertaking research: the experience of novice researchers". British Journal of Nursing 17: 920-923.

9. G Lancaster, M Campbell, S Eldridge, A Farrin, M Marchant, et al. (2010) "Trials in primary care: statistical issues in the design, conduct and evaluation of complex interventions". Statistical Methods in Medical Research 19: 349-377.

10. L Matowe, C Leister, C Crivera, J Korth Bradley (2003) "Interrupted Time Series Analysis in Clinical Research". The Annals of Pharmacotherapy 37: 1110-1116.

11. World Health Organization (2010) "TELEMEDICINE Opportunities and Developments in Member States. Report on the Second Global Survey on eHealth". Geneva.

12. Invest NI and DHSSPS (2011) "Connected Health and Prosperity Memorandum of Understanding Between The Department of Health, Social Services and Public Safety, and Invest Northern Ireland". Department of Health, Social Services and Public Safety.

13. MRC (2006) "Developing and Evaluating Complex Interventions: New Guidance". United Kingdom: Medical Research Council.

14. M Handley, D Schillinger, S Shiboski (2011) "Quasi-Experimental Designs in Practice-based Research Settings: Design and Implementation Considerations". Journal of the American Board of Family Medicine 24: 589-596.
15. C Renders, G Valk, S Griffin, E Wagner, J van Eijk, et al. (2000) "Interventions to improve the management of diabetes mellitus in primary care, outpatient and community settings (Review)". The Cochrane Library 4

16. F Zhang, AK Wagner, SB Soumerai, D Ross Degnan (2009) "Methods fo estimating confidence intervals in interrupted time series Abstract analyses of health interventions". Journal of Clinical Epidemiology 62: 143-148.

17. C Ramsay, L Matowe, R Grilli, J Grimshaw, R Thomas (2003) "Interrupted Time Series Designs in Health Technology Assessment: Lessons From Two Systematic Reviews of Behavior Change Stratigies". International Journal of Technology Assessment in Health Care 19: 613-623

18. (2015) "Integrated Research Application System".

19. NHS Health Research Authority (2015) "Research Ethics Committee: Consent and Participants Information".

20. AccessNI Criminal Check Check (2014) "NIdirect government services".

21. LK McCormick, M Crawford, R H Anderson, J Gittelsohn, B Kingsley, et al (1999) "Recruiting Adlescents into Qualitative Tobacco Research Studies: Experiences and Lessons Learned". Journal of School Health 69: 95-99.

22. E Fairbanks, S Shah, MH Wilde, MV McDonald, J Brasch, et al. (2014) "Successful recruitment methods in the community for a two-site clinical trial". Applied Nursing Research 23: 254-257.

23. KD Jones, AC Reiner (2010) "A multistep recruitment strategy to a participantintensive clinical trial”. Applied Nursing Research 23: 227-232.

24. JK Payne, CC Hendrix (2010) "Clinical trial recruitment challenges with older adults with cancer". Applied Nursing Research 23: 233-237.

25. "NHS funding and finances," 2015.

26. Z Lu, J Su (2010) "Clinical data management: Current status, challenges, and future directions from industry perspectives". Journal of Clinical Trials 2: 93-105.

27. (2015) "Review: Clinical trials data collection: how big data could be a big challenge".

28. S de Lusignan, C van Weel (2005) "The use of routinely collected computer data for research in primary care: opportunities and challenges". Family Practice Advance Access 23: 253-263.

29. R Richesson, P Nadkarni (2011) "Data standards for clinical research data collection forms: current status and challenges". Journal of the American Medical Informatics Association 18: 341-346.

30. MX Patel, V Doku, L Tennakoon (2003) "Challenges in recruitment of research participants". Advances in Psychiatric Treatment 9: 229-238.

31. JN Weinstein, RA Deyo (2000) "Clinical Research: Issues in Data Collection". SPINE 25: 3104-3109

32. H Mushcab, G Kernohan, J Wallace, S Martin (2015) "Web-Based Remote Monitoring Systems for Self-Managing Type 2 Diabetes: A Systematic Review". DIABETES TECHNOLOGY \& THERAPEUTICS 17: 498-509.

33. "Ulster Hospital". http//www.setrusthscni.net/2024.htm)accesses on 10 of July 2015 